\title{
A Dimensão Religiosidade/Espiritualidade na Prática Clínica: Revisão Integrativa da Literatura Científica
}

\author{
Vivian Fukumasu da Cunha* (D) \& Fabio Scorsolini-Comin (D) \\ ${ }^{1}$ Universidade de São Paulo, Ribeirão Preto, SP, Brasil
}

\begin{abstract}
RESUMO - Este estudo apresenta uma revisão integrativa sobre como a religiosidade/espiritualidade (R/E) está presente na literatura científica acerca da prática clínica em Psicologia. As buscas nas bases LILACS, SciELO, PePSIC, MEDLINE/ PubMed e PsycINFO (janeiro 2010/março 2016) recuperaram 50 produções predominantemente qualitativas e publicadas em periódicos internacionais. Evidenciou-se que a R/E vem sendo incorporada nas noções de saúde, cuidado e integralidade, sendo reconhecida como dimensão que possui aspectos culturais que devem ser considerados na clínica. A literatura apresenta alguns protocolos e estratégias que podem ser empregados na prática clínica como forma de integrar a R/E ao cuidado oferecido. Sugere-se que os pesquisadores brasileiros continuem investigando o tema, visando a fornecer subsídios para práticas éticas e inovadoras quanto às dificuldades enfrentadas pelos psicoterapeutas e recém-formados.
\end{abstract}

PALAVRAS-CHAVE: espiritualidade, religiosidade, psicologia clínica, psicoterapia, psicoterapeuta

\section{The Religiousness/Spirituality Dimension in Clinical Practice: An Integrative Review of the Scientific Literature}

\begin{abstract}
This study presents an integrative review on how religiousness/spirituality $(\mathrm{R} / \mathrm{S})$ is present in the scientific literature on clinical practice in Psychology. The searches in LILACS, SciELO, PePSIC, MEDLINE/PubMed and PsycINFO databases (January 2010/March 2016) showed 50 predominantly qualitative productions published in international journals. It was evidenced that $\mathrm{R} / \mathrm{S}$ has been incorporated into the notions of health, care and integrality, being recognized as a dimension with cultural aspects that must be considered in clinical practice. The literature presents some protocols and strategies that can be used in clinical practice as an alternative to integrate R/S to the care offered. It is suggested that Brazilian researchers continue to investigate the topic in order to offer ethical and innovative practices regarding the difficulties faced by psychotherapists and newly graduates.
\end{abstract}

KEYWORDS: spirituality, religiousness, clinical psychology, psychotherapy, psychotherapist

A produção científica relacionada à religiosidade/ espiritualidade $(\mathrm{R} / \mathrm{E})$ vem se tornando cada vez mais expressiva na contemporaneidade, sendo associada a dimensões como saúde, cuidado, integralidade e humanização (Dalgalarrondo, 2007; Freitas, 2014; Hill et al., 2000; Marques, 2010). Apesar das especificidades de cada uma das noções - religiosidade e espiritualidade -, e das tensões epistemológicas muitas vezes presentes entre a Psicologia da Espiritualidade e a Psicologia da Religião (Paiva, 2005), adotaremos, neste estudo, a terminologia combinada, ou seja, a R/E, assumindo a complexidade dessas noções e das variadas definições disponíveis na literatura, com foco na associação entre esses dois fenômenos. Além disso, o termo combinado tem sido encontrado na literatura abordando suas relações e não os fenômenos em si (Alminhana \& Moreira-Almeida, 2009; Genaro Junior, 2011; Henning-Geronasso \& Moré, 2015; Oliveira \& Junges, 2012), assim como proposto na presente revisão.

A R/E é uma dimensão que faz parte do conceito multidimensional de saúde proposto pela World Health

Apoio: $\mathrm{CNPq}$

*E-mail: vivianfcunha@gmail.com

- Submetido: 22/09/2016; Revisado: 16/01/2018; Aceito: 19/02/2018. 
Organization - WHO (1998). No esteio dessa consideração, pesquisas apresentadas na revisão de literatura realizada por Koenig (2012) sugerem que a R/E pode influenciar a saúde mental por meio de vários mecanismos: pode promove recursos de enfrentamento de situações de estresse, aumentar a frequência de emoções positivas e diminuir a probabilidade de depressão e transtorno de ansiedade, por exemplo. Além disso, segundo o autor, pessoas mais espiritualizadas ou religiosas se adaptam melhor e mais rapidamente a problemas de saúde se comparadas àquelas pouco ou nada espiritualizadas ou religiosas. Pessoas religiosas também se mostram menos vulneráveis ao uso de drogas, ao divórcio, ao envolvimento com o crime e ao suicídio (Seligman, 2009). A R/E vem sendo relacionada a sentimentos como os de maior propósito na vida, otimismo, generosidade para com os outros e expressão de gratidão (Koenig et al., 2014), aspectos estes que podem sugerir um melhor ajustamento das circunstâncias adversas na vida e também contribuir para melhores condições de saúde.

Em uma pesquisa realizada com jovens entre 18 e 24 anos da cidade de Pelotas, no Rio Grande do Sul, essa dimensão apresentou associações positivas com o bem-estar subjetivo, bem-estar espiritual e bem-estar psicológico, sendo que quanto maior a $\mathrm{R} / \mathrm{E}$ do sujeito, maior o bem-estar avaliado (Souza et al., 2012). Outras associações positivas aparecem entre $\mathrm{R} / \mathrm{E}$, qualidade de vida e coping religioso-espiritual (Panzini, Rocha, Bandeira, \& Fleck, 2007) e entre R/E e saúde mental, com menor prevalência de depressão e ansiedade, menor tempo de remissão da depressão após o tratamento e menor taxa de suicídio, além de outras evidências (Koenig et al., 2014; Lucchetti, Granero, Bassi, Latorraca, \& Nacif, 2010; Smith, McCullough, \& Poll, 2003).

Apesar desses indícios positivos envolvendo a $\mathrm{R} / \mathrm{E}$, cabe destacar que estes não podem ser generalizados a todas as situações e pessoas. A literatura científica também explora efeitos negativos da $\mathrm{R} / \mathrm{E}$ sobre a saúde das pessoas, como recusa à busca de tratamentos formais e negligência em relação a tratamentos prescritos por profissionais de saúde, bem como repercussões que colocam a R/E como única solução para determinados problemas, por exemplo, desconsiderando outros conhecimentos produzidos e formas de atenção que poderiam ser empregados e terem efeitos positivos (Koenig, 2005; Lucchetti et al., 2010).

$\mathrm{Na}$ interface entre Psicologia, R/E e Saúde Mental, o Manual Diagnóstico e Estatístico de Transtornos Mentais (DSM-V) (American Psychiatric Association - APA, 2014) traz a categoria "Problemas Religioso ou Espiritual", abarcando questões de ordem religiosa ou espiritual que emergem no contexto da prática clínica, colocando em destaque problemáticas que buscam na psicoterapia um possível tratamento e uma compreensão para as suas ocorrências, como a perda ou questionamento da fé. As relações entre esses fenômenos espirituais e religiosos e a saúde mental ainda oferecem constantes reflexões por parte de pesquisadores, psicoterapeutas e psiquiatras diretamente envolvidos nessa temática, de modo que tal discussão se mostra recorrente (Silva \& Reichow, 2016).

Visando a ampliar tal debate e divulgar pesquisas conduzidas nessa interface, o Conselho Regional de Psicologia de São Paulo (CRP-SP) publicou, em 2016, a coleção "Psicologia, laicidade e as relações com a religião e a espiritualidade". Essa publicação, fruto de diversos debates organizados e fomentados pelo CRP-SP, apresenta a necessidade de promover uma formação e discussão mais adequadas dos profissionais de Psicologia quanto ao tema. De acordo com Freitas (2014), psicólogos recém-formados reconhecem a presença de questões religiosas e espirituais no discurso e na experiência de seus pacientes/clientes, mas se sentem despreparados para lidar com tal realidade, demonstrando receios de virem a incorrer em problemas de cunho ético, denunciando uma lacuna em seus aprendizados. A ausência do tema na formação em Psicologia também foi exposta pelos participantes da pesquisa de HenningGeronasso e Moré (2015).

No Brasil, o Sistema de Conselhos de Psicologia reconhece a importância dessa dimensão na constituição de subjetividades, em que se faz fundamental o estabelecimento de um diálogo entre os conhecimentos acumulados pela $\mathrm{R} / \mathrm{E}$ e pela Psicologia, possibilitando maior conhecimento das interfaces estabelecidas entre elas (Conselho Federal de Psicologia, 2013). Considerando que a psicoterapia é uma das atividades mais desempenhadas pelos psicólogos e que o Brasil representa um contexto social fortemente influenciado por tradições religiosas, crenças e experiências místicas, em um panteão diverso e multifacetado (Bairrão, 2004), a relação entre $\mathrm{R} / \mathrm{E}$ e prática clínica passa a ser evocada de modo mais expressivo.

No campo acadêmico, Freitas (2014) destaca que em pesquisa com professores de psicopatologia em Brasília, realizada por Carneiro (2008), os profissionais têm evitado tratar do assunto em sala de aula, embora reconheçam serem muito comuns as perguntas dos alunos em torno das relações da R/E com a saúde/doença mental. É salientado também que o desconhecimento de uma literatura consistente e o receio de que a discussão se encaminhe para questões metafísicas ou relacionadas a crenças religiosas específicas, que não encontram respaldo científico e que fogem dos objetivos da disciplina, são os principais motivos para não se falar no assunto. Já na perspectiva de 136 universitários de uma universidade privada de Porto Alegre, $83,8 \%$ manifestaram ter interesse pelo assunto e $77,9 \%$ desejam discutir o assunto em sua formação (Costa et al., 2008), sugerindo uma demanda e necessidade para subsidiar o trabalho e conhecimentos do futuro profissional. Tal dificuldade pode ser explicada, ainda, por um cuidado ético em não induzir os pacientes/clientes a qualquer credo. Como apresentado por Scorsolini-Comin (2015a), as recomendações éticas existem no sentido de que o profissional não "influencie" ou "induza" seu paciente/ cliente a determinados posicionamentos religiosos ou 
práticas espirituais, o que não significa desconsiderar ou negligenciar essa dimensão na escuta clínica. No entanto, como forma de se resguardar de possíveis sanções éticas, a dimensão da $\mathrm{R} / \mathrm{E}$ pode ser considerada um tabu na prática clínica, demandando maior cuidado em seu manejo, e até mesmo afastando os profissionais dessa discussão.

No campo internacional, notadamente nos Estados Unidos e Canadá, focaliza-se mais a relação de terapeutas com a temática, apontando para a necessidade de maiores investigações e estudos sobre questões éticas envolvidas, cuidados para abordar o assunto, necessidade de treinamento apropriado e supervisões para lidar com as dificuldades que o tema oferece (Daniels \& Fitzpatrick, 2013; Leighton, 2014; Lomax \& Carlin, 2015). Assim, a necessidade de discutir o tema na formação já se apresenta como um mote para pesquisas no exterior, em contraposição ao movimento ainda velado que se observa na literatura nacional. Também se destaca a relevância dessa discussão no âmbito clínico, haja vista as associações desse tema com diversas patologias e transtornos, revelando a necessidade dos pacientes em relação ao assunto (Koenig, 2012).
Frente a esse crescente interesse no tema da $R / E$, destaca-se que a clínica pode ser um espaço em que o psicólogo, enquanto facilitador do processo terapêutico, pode reconhecer essa dimensão como parte da relação psicoterápica (Oliveira \& Junges, 2012), devendo estar atento tanto às associações positivas quanto negativas que podem emergir quando a $\mathrm{R} / \mathrm{E}$ é considerada na prática clínica. Estudos têm sugerido que, implícita ou explicitamente, não há como desconsiderá-la no "fazer" clínico (Pargament, 2007; Vandenberghe, Prado, \& Camargo, 2012), sendo que a $\mathrm{R} / \mathrm{E}$ do paciente/cliente deve ser alvo de inquérito no atendimento clínico (Lucchetti et al., 2010), permitindo uma visão mais ampla acerca do sujeito e a consideração de sua integralidade. $\mathrm{O}$ reconhecimento da $\mathrm{R} / \mathrm{E}$ do próprio clínico também vem sendo alvo de investigações (ScorsoliniComin, 2015a), acrescentando outros elementos à discussão.

A partir desse panorama e tendo como norteadora a necessidade de constante atualização de conhecimentos nesse campo, o objetivo deste estudo é apresentar uma revisão integrativa sobre como a $\mathrm{R} / \mathrm{E}$ está presente na literatura científica acerca da prática clínica em Psicologia.

\section{MÉTODO}

Trata-se de uma revisão integrativa da literatura científica, que tem como uma de suas metas a busca de evidências consolidadas para a prática baseada em evidências - PBE. Foram adotados os procedimentos propostos por Mendes, Silveira e Galvão (2008): (1) identificação do tema e da questão norteadora; (2) estabelecimento de critérios de inclusão/exclusão; (3) categorização dos estudos; (4) avaliação dos estudos; (5) interpretação dos resultados; (6) síntese do conhecimento. A revisão também foi conduzida a partir do formulário internacional para estudos de revisão sistemática e metanálises, o PRISMA - Preferred Reporting Items for Systematic Reviews and Meta-Analyses, que contém 27 itens de verificação para avaliação de revisões de literatura (Moher, Liberati, Tetzlaff, Altman, \& The PRISMA Group, 2009). Esse protocolo foi empregado tanto para nortear a inclusão dos estudos na revisão (avaliação das evidências incluídas) quanto para orientar o delineamento do presente estudo em termos de sua execução e escrita.

O processo de seleção e a categorização dos estudos foram realizados por dois juízes independentes. Nos casos de discordância, um terceiro juiz foi acionado. Seguindo protocolos internacionais para os estudos de revisão integrativa, a questão norteadora foi definida a partir do método PICO ( $\mathrm{P}=$ participantes; $\mathrm{I}=$ intervenção; $\mathrm{C}=$ comparação; $\mathrm{O}=$ resultado/desfecho) (Santos, Pimenta, \& Nobre, 2007), ficando redigida desse modo, a partir de adaptações pertinentes à área na qual se situa este estudo: como a R/E (P) está presente na literatura científica $(\mathrm{O})$ que trata da prática clínica de Psicologia (I)? Como o objetivo não foi estabelecer uma comparação entre cenários de atuação ou entre áreas do conhecimento, o critério C não foi empregado na redação da pergunta, o que já é previsto na metodologia PICO para alguns casos.

\section{Bases Indexadoras e Unitermos Empregados}

A fim de ampliar as fontes de evidência, foram consultadas as bases/bibliotecas LILACS, SciELO, PePSIC, MEDLINE/PubMed e PsycINFO. Os unitermos de busca utilizados foram consultados previamente na Terminologia em Psicologia da BVS-Psi, DeCS (Descritores em Ciências da Saúde), no MeSH (Medical Subject Headings) e no Term Finder da APA. De modo complementar, foram acrescidas palavras-chaves a partir de consultas realizadas a artigos publicados sobre o tema e que não tinham como unitermos aqueles presentes nesses índices. Os unitermos empregados foram "Espiritualidade/Spirituality" OR "Religiosidade/ Religiousness" OR "Crença religiosa/Religious belief" OR "Religioso/Religious" OR "Religião/Religion" OR "Prática religiosa/Religious practice" combinados a partir do operador booleano "and" com "Psicologia Clínica/ Clinical Psychology" OR "Processo terapêutico/Therapeutic process" OR "Psicoterapia/Psychotherapy" OR "Terapia/ Therapy" OR "Psicólogo/Psychologist".

\section{Critérios de Inclusão e de Exclusão}

Foram incluídos artigos científicos completos, indexados nas bases selecionadas, nos idiomas português e inglês, publicados entre janeiro de 2010 e março de 
2016, que tiveram a temática relacionada com o objetivo do estudo e possibilitaram responder à questão norteadora pré-definida. $\mathrm{O}$ recorte temporal buscou a inclusão de evidências mais recentes, de modo a compreender o status da produção contemporânea sobre o tema. Foram excluídas outras publicações (teses, dissertações, monografias, resenhas, cartas, editoriais, notícias, obituários, livros e capítulos), artigos fora do período de publicação estabelecido, incompletos e em outros idiomas que não português e inglês. Também foram excluídos aqueles que se relacionaram com o tema, mas que não responderam à questão norteadora ou que abordaram a temática apenas de modo tangencial.

\section{Procedimento}

O levantamento dos dados foi realizado no mês de abril de 2016. Os unitermos foram combinados e os registros encontrados foram lidos em termos de título e o resumo. Apenas os artigos relacionados ao tema e em consonância com os critérios estabelecidos foram selecionados. Esse procedimento foi realizado por dois juízes independentes, ambos com formação em Psicologia e com treinamento e familiaridade com o tema e os procedimentos da revisão. As discordâncias foram analisadas por um terceiro juiz, também com ampla experiência na área. Os artigos repetidos foram contabilizados uma única vez. Os artigos selecionados a partir da leitura dos títulos e resumos foram recuperados para leitura na íntegra. Novamente foram aplicados os critérios de inclusão e exclusão, tomando como referência os textos completos. Foram recuperados e analisados na íntegra os estudos que restaram a partir desse processo de leitura e análise, compondo o corpus final.

\section{Análise de Dados}

O corpus foi organizado em uma planilha de Excel, destacando, para cada artigo recuperado na amostra final, os seguintes aspectos necessários para a identificação do perfil das publicações: título, autores, instituição de origem dos autores, ano de publicação, periódico, objetivo, método/tipo de estudo, amostra, instrumentos, principais resultados, principais conclusões, limites e potencialidades/ contribuições para novos estudos.

A apresentação da revisão/síntese do conhecimento pautou-se nas recomendações do sistema PRISMA a partir dos critérios preconizados em sua lista de verificação (Moher et al., 2009). O PRISMA foi utilizado para avaliar as características de redação do estudo e não necessariamente sua qualidade metodológica, sendo uma diretriz que tem como objetivo ajudar autores a melhorarem a qualidade do relato dos dados das revisões. A partir do delineamento do perfil de produções, apresentado na seção de Resultados, os artigos foram analisados na íntegra para a construção de categorias, de modo a responder à questão norteadora e atender aos objetivos específicos da revisão.

\section{RESULTADOS}

Os processos de busca, seleção e recuperação de evidências estão sumarizados no fluxograma (Figura 1). A maior parte das investigações que compuseram o corpus foi qualitativa $(88 \%)$, seguida por investigações quantitativas e mistas, com $6 \%$ cada. Os artigos são predominantemente em idioma inglês $(86 \%)$ e o restante $(14 \%)$ em português. O ano de maior evidência foi 2010 , com $24 \%$ das publicações, seguido pelos anos 2013, 2014 e 2015, com 16\% cada um deles.

Pela análise do país de origem do primeiro autor de cada artigo recuperado, observou-se a presença de produções oriundas do Brasil, Estados Unidos, Canadá, África do Sul, França, Egito, Noruega, Sri Lanka, Israel, Chipre, Irã e Reino Unido. Os Estados Unidos, isoladamente, representaram $52 \%$ da amostra. O periódico que mais apareceu entre as publicações foi Spirituality in Clinical Practice (10\%), seguido por Psychology of Religion and Spirituality (8\%) e por Professional Psychology: Research and Practice, Journal of Religion and Health e Cognitive and Behavioral Practice, com 6\% cada. Há que se considerar, portanto, que as comunicações sobre a $\mathrm{R} / \mathrm{E}$ ainda parecem estar centradas em periódicos específicos e com políticas editoriais especializadas nesse campo, que pode tornar a produção um nicho, com maior dificuldade de ser veiculada em revistas generalistas das áreas de Psicologia, Medicina, Enfermagem ou mesmo de Saúde Mental ou de Psicologia Clínica. Observa-se um movimento de encaminhamento dessas produções a revistas que, possivelmente, possuem políticas editoriais mais centralizadas nesses fenômenos, o que pode ser um indício importante para localizar essa produção, mas diminui o seu diálogo com outras temáticas no campo da Saúde e da Psicologia.

As investigações qualitativas eleitas nessa revisão se apresentaram a partir de diversos estudos de caso, formulação de teorias e abordagens específicas com ilustração de caso (Karekla \& Constantinou, 2010), relatos de experiência profissional (Genaro Junior, 2011) e investigações exploratórias com profissionais (HenningGeronasso \& Moré, 2015; Oliveira \& Junges, 2012), e empregaram instrumentos como entrevistas e questionários abertos ou semiestruturados (Zenkert, Brabender, \& Slater, 2014). Os estudos quantitativos utilizaram escalas e questionários como o The Spiritual Transcendence Index (Cornish, Wade, \& Knight, 2013), Religious Commitment Inventory - 1 (RCI-10), Revised Dyadic Adjustment Scale - 4 (RDAS-4), Working Alliance Inventory (WAI-ST) e o Client 


\subsection{8 registros encontrados}

PePSIC (n=29), SciELO (n=75), LILACS ( $\mathrm{n}=348)$, MEDLINE $(n=1.564)$ e PsycINFO $(n=4.812)$

\subsection{6 registros nos anos de 2010 até março de 2016}

PePSIC ( $n=16)$, SciELO ( $n=41)$, LILACS $(n=164)$, MEDLINE $(n=367)$ e PsycINFO $(n=558)$

\section{8 foram excluídos pela leitura de títulos e resumos}

- Não respondem a questão norteadora porque $(n=944)$ :

(a) Se distanciam do tema (ex. situações médicas e doenças, uso de substâncias, contextos sociais e interventivos aleatórios, relação com a famíla e adoecimento, cura religiosa ou místicas, outros) ( $\mathrm{n}=397$ )

(b) Apresenta a temática superficialmente ou de maneira secundária em relação com outras variáveis (ex. enfrentamento, esperança, abuso religioso, altruísmo, terapia ocupacional, arte terapia, benefício e aderência à tratamentos médicos, outros $)(\mathrm{n}=290)$

(c) Sujeitos de pesquisa não são psicólogos ou psicoterapeutas (ex. médicos, enfermeiros, pacientes/clientes, estudantes, pedagogo, outros) ( $\mathrm{n}=114)$

(d) Investiga a eficácia de intervenções ou instrumentos $(\mathrm{n}=18)$

(e) Aborda a temática teoricamente, não oferece a perspectiva ou experiência do profissional $(n=125)$

- Revisões em torno do tema (ex. crítica, da literatura) $(n=19)$

- Publicações que não artigos (ex. livro, introdução, entrevista) (n=10)

- Outros Idiomas $(\mathrm{n}=70)$

- Repetidos ( $\mathbf{n = 4 5 )}$

58 artigos foram incluídos para leitura na íntegra

08 artigos não foram encontrados (textos completos)

\section{Corpus final: $\mathbf{N}=\mathbf{5 0}$}

PePSIC $(n=1)$, SciELO $(n=3)$, LILACS $(n=3)$, MEDLINE $(n=10)$ e PsycINFO $(n=33)$

Figura 1. Fluxograma de busca, seleção e composição do corpus.

Satisfaction Questionnare (CSQ) (Hook, Worthington Jr., Davis, \& Atkins, 2013).

Os principais objetivos dos artigos foram investigações ou ilustração de aplicação de uma abordagem ou teoria na prática (Abu-Raiya, 2015; Rich, 2012), experimentação de ferramentas e estratégias que vêm sendo desenvolvidas em relação à temática (Daniels \& Fitzpatrick, 2013; Leighton, 2014; Walker, Reese, Hughes, \& Troskie, 2010) e identificação de características, barreiras, conhecimentos e influências dos terapeutas na prática profissional (Brown, Elkonin, \& Naicker, 2013). Nas abordagens teóricas adotadas pelas propostas, uma variedade de perspectivas e técnicas foi encontrada, como a terapia cognitivo-comportamental (Dein, 2013), a terapia de aceitação e compromisso (Karekla \& Constantinou, 2010), a humanista (Keeling, Dolbin-MacNab, Ford, \& Perkins, 2010), a psicodinâmica (Daniels \& Fitzpatrick, 2013), a cognitiva (Zoysa, 2011), a psicanálise winnicottiana (Genaro Junior, 2011), a psicanálise contemporânea (Aten,
Mangis, \& Campbell, 2010), a psicoterapia corporal (Gottwald, 2014), a etnopsiquiatria (Zajde, 2011), a terapia do bem-estar (Sperry, 2010), a transpessoal (Rich, 2012), a hipnose (Neubern, 2010a), bem como novas e específicas abordagens adaptadas e criadas para determinado contexto religioso/cultural, como em latinos ou muçulmanos (AbuRaiya, 2015).

Foram identificadas intervenções psicoterápicas individuais (Neubern, 2010b), familiares (Khodayarifard, \& Fatemi, 2013), grupais (Wade, Post, Cornish, Vogel, \& Runyon-Weaver, 2014) e de casal (Hook et al., 2013), em contextos de consultório privado (Gottwald, 2014), em clínicas psicológicas filiadas a universidades (Zajde, 2011) e hospitais psiquiátricos (Stalsett, Engedal, \& Austad, 2010). Os pacientes/clientes podiam ser divididos desde entres os que sofriam de doenças crônicas, como câncer (Karekla \& Constantinou, 2010) e transtornos mentais diversos (esquizofrenia, transtorno de ansiedade generalizada, depressão) (Barrera, Zeno, Bush, Barber, \& Stanley, 
2012) e aqueles que apresentavam dificuldades cotidianas, relacionais, de adaptação às mudanças ou que passaram por alguma situação traumática (por exemplo, abusos e imigração) (Walker et al., 2010; Zajde, 2011).

A partir da explicitação do perfil dessas publicações, a etapa "Discussão", a seguir, será orientada por três categorias, construídas em resposta à questão norteadora e tendo em vista os resultados disponíveis no corpus, analisados de modo integrativo: (a) a consideração da $\mathrm{R} / \mathrm{E}$ nas noções de saúde, cuidado e integralidade; (b) aspectos culturais da $\mathrm{R} / \mathrm{E}$ e a prática clínica; (c) R/E e prática clínica: estratégias, intervenções e protocolos.

\section{DISCUSSÃO}

\section{Categoria I:A Consideração da R/E nas Noções de Saúde, Cuidado e Integralidade}

A dimensão R/E é compreendida como algo inerente ao ser humano em todas as produções recuperadas. Nessa acepção, um modelo mais integralista, que incorpora o aspecto espiritual do cuidado, tornou-se mais amplamente aceito como um quadro para a compreensão das necessidades dos pacientes e suas famílias, entendendo o adoecimento pela complexa interação de fatores biológicos, sociais, psicológicos e espirituais (Richardson, 2014). Outros estudos dessa revisão reportaram essa postura, considerando a $\mathrm{R} / \mathrm{E}$ como parte integrada à condição humana e reconhecendo sua influência na saúde (Aten et al., 2010; Gottwald, 2014; Rich, 2012), o que se torna lícito em função da noção multidimensional de saúde proposta pela OMS (WHO, 1998).

Dal-Farra e Geremia (2010) consideram que partir do ponto de vista de um ser humano na sua totalidade, considerando os diversos aspectos de sua individualidade e de sua relação com o meio, amplia-se e enriquece-se a relação com o paciente. Isso conduz a novos horizontes preventivos, diagnósticos e terapêuticos, possibilitando aos profissionais da saúde atuarem como seres humanos também em sua totalidade, o que é, cientificamente, terapêutico, por influir em todas as etapas do processo do cuidar. Os autores acrescentam que, partindo da premissa referente à interconexão entre mente e corpo e aos reflexos do bem-estar sobre a saúde, a adoção de estratégias que integrem os amplos domínios da subjetividade humana, incluindo a $\mathrm{R} / \mathrm{E}$, representa um ponto fundamental nas práticas pedagógicas do ensino na área da saúde. Nesse sentido, temas relacionados à $\mathrm{R} / \mathrm{E}$ devem estar presentes na formação e compreensão dos profissionais da Saúde, o que nem sempre é a realidade. Essa necessidade também é deflagrada em produções brasileiras, a exemplo da coleção sobre religião e laicidade publicada pelo CRP-SP (2016).

Alguns artigos recuperados revelaram que os profissionais consideram essa dimensão importante e estão dispostos a integrar a $\mathrm{R} / \mathrm{E}$ em suas práticas, mas muitas vezes não se sentem competentes o bastante para abordar o assunto, denunciando uma falha em seus treinamentos ou na graduação e sinalizando a necessidade de maior atenção ao tema na formação (Brown et al, 2013; Henning-Geronasso \& Moré, 2015; Keeling et al., 2010). Cavalheiro e Falcke (2014) corroboram esses achados e ainda apontam que a formação no curso de Psicologia, tendo como referência estudantes do Estado do Rio Grande do Sul, contribui para que a $\mathrm{R} / \mathrm{E}$ dos formandos seja menor do que a de calouros do curso. Essas autoras atribuem esse resultado ao contato com a racionalidade e cientificidade do curso e da possível influência da vertente psicanalítica sobre o perfil dos estudantes, sendo que formandos que se identificam com a psicanálise também apresentaram menores índices de R/E se comparados com outras abordagens.

De fato, nesta própria revisão também foram encontrados artigos com críticas à psicanálise. Para esses críticos, a psicanálise não considera de modo adequado as minorias raciais e étnicas, em contraposição à construção de uma atenção mais multicultural e com destaque para a diversidade presente nos currículos de cursos de Aconselhamento e Psicologia (Ciclitira \& Foster, 2012). Pargament (2007) relata que Sigmund Freud criticou a religião, anunciando que esta seria apenas uma sensação ilusória de segurança e, nesse caso, por pior que seja, enfrentar a realidade como é parece ser a melhor solução. No entanto, Lomax e Carlin (2015) acreditam que isso deixaria de lado as relações positivas que a R/E pode apresentar, como, por exemplo, confortar em situações de perda. Ressalta-se que se deve manter um olhar cuidadoso ao fazer considerações a respeito da psicanálise ou mesmo das críticas a Freud, pois também foram encontrados, a partir desta revisão, artigos que usaram a psicanálise como referencial. Pesquisas recuperadas no corpus e de base psicanalítica destacaram que a R/E pode ser parte importante para compreender o contexto cultural religioso/espiritual do paciente/cliente (Aten et al., 2010), uma maneira de conhecer como a pessoa se apresenta no mundo (Genaro Junior, 2011) e um assunto que pode ampliar a conexão com o paciente/cliente na criação de vínculo para realizar o trabalho (Jones, 2014). Até mesmo pela variedade como as diferentes abordagens são assumidas e trabalhadas nesses estudos, deve-se considerar com parcimônia tais apontamentos, primando sempre pelo diálogo positivo entre referenciais na ampliação do conhecimento científico sobre a R/E.

Observa-se que a $\mathrm{R} / \mathrm{E}$ vem sendo considerada importante para a relação com o paciente/cliente e no processo de saúde/doença, no qual um modelo mais integralista de cuidado reconhece a R/E como componente dessa relação, sensibilizando os profissionais em uma visão holística e humanizada. Torna-se claro na prática clínica que, na maioria 
das vezes, não é possível fragmentar o paciente/cliente em diversas dimensões, pois todas são interligadas (Lucchetti et al., 2010). Torna-se importante pensar as implicações disso para a prática profissional e rever a necessidade de reformulação dos paradigmas norteadores da ciência psicológica (Cavalheiro \& Falcke, 2014), já que a inclusão de disciplinas que abordam questões da $\mathrm{R} / \mathrm{E}$ permite tratar esse tema em sua amplitude, por meio de suas interrelações com outras temáticas (Dal-Farra \& Geremia, 2010). É importante considerar que o olhar para a $\mathrm{R} / \mathrm{E}$ na prática clínica não deve focar apenas as disciplinas, mas também a formação em serviços, nas supervisões clínicas e em outros fóruns de discussão.

Como exemplo de situações em que a R/E aparece, podese pensar no diagnóstico diferenciado entre os transtornos mentais de conteúdo religioso e as experiências espirituais que fazem parte da vivência cultural dos sujeitos (Menezes Jr \& Moreira-Almeida, 2009), ou como um fator de suporte para lidar com situações de estresse e adversidades, como o coping religioso/espiritual (Panzini \& Bandeira, 2007). Sensibilizar os estudantes de graduação para R/E, assim como os demais profissionais da Psicologia e da área de Saúde, é caminhar em direção ao que é proposto pela WHO (1998). Considerar essa dimensão como parte do conceito multidimensional de saúde reflete o que foi recuperado pelo corpus, evidenciando que R/E não é uma característica dissociada ou deixada de lado nos atendimentos, mas que não precisa ser demasiadamente exaltada.

\section{Categoria 2: Aspectos Culturais da R/E e a Prática Clínica}

Alguns artigos consideraram a R/E como característica fundamental da cultura dos indivíduos. Nestes, foi destacado o aspecto espiritual/religioso de algumas populações e como essas características influenciam hábitos e costumes, levantando, também, a necessidade de sensibilização de minorias étnicas e enunciando a importância de não julgar a veracidade ou contestar possíveis crenças (Abu-Raiya \& Pargament, 2010; Aten et al., 2010; Ciclitira \& Foster, 2012; Daniels \& Fitzpatrick, 2013; Zajde, 2011). Essa realidade vai ao encontro do fato de que cada vez mais a $R / E$ vem sendo percebida como parte do processo cultural e, se bem interpretada e avaliada, pode fornecer informações essenciais para a compreensão de questões atuais do paciente/cliente, como métodos para tratá-lo adequadamente (Allmon, 2013). Nesse sentido, estudos com populações mulçumanas e latinas, além de outras, representaram muito bem a caracterização, influência e destaque que a R/E ganha na vida desses sujeitos, denunciando a importância de se evitar estereótipos, generalizações ou uma visão simplista (Abu-Raiya \& Pargament, 2010).

Ao optarmos pelo olhar científico da clínica psicológica, é preciso que o profissional compreenda de que modo as crenças constituem a pessoa, seu modo de ser e funcionar
(Scorsolini-Comin, 2015a), bem como suas ancoragens na coletividade, em suas comunidades de referência, em busca de uma escuta que não é sempre individual. Assim, é necessário colocar-se disponível para refletir sobre culturas diferentes, em uma consideração positiva incondicional sobre essa realidade. Nesse processo de reconhecimento de si nas práticas culturais consideradas diferentes ou distantes, $\mathrm{o}$ profissional deve estar atento à complexidade inerente dessa tarefa, haja vista que nem sempre podemos controlar o modo como nossos valores sociais são construídos e incorporados em nossas práticas e formas de ser e de nos relacionarmos (Scorsolini-Comin, 2015b).

Os seres humanos, prenhes de cultura, corporificam práticas, ritos, crenças, afetos, formas de compreender e significar o mundo, e com os profissionais isso não é diferente (Scorsolini-Comin, 2015b). Em alguns artigos recuperados, a $\mathrm{R} / \mathrm{E}$ do próprio psicoterapeuta foi reconhecida como um aspecto que influencia a relação psicoterápica, levando o profissional a experienciar conflitos de sentimentos e de identificação, bem como a ser tendencioso ou a elevar a quantidade de intervenções sobre o tema, provocando incômodos nos pacientes/clientes (Brown et al., 2013; Cornish, Wade, \& Knight, 2013; Hook et al., 2013; Keeling et al., 2010; Vandenberghe et al., 2012; Zenkert et al., 2014). Considerações semelhantes são evidenciadas com outros profissionais da saúde como, por exemplo, os médicos (Lucchetti et al., 2010). Diante disso, em consonância com a literatura, sinaliza-se que o psicoterapeuta deve estar atento à sua própria $\mathrm{R} / \mathrm{E}$, compreendendo de que modo isso está presente em sua vida e em sua profissão, permitindo uma relação terapêutica ética, na qual os pacientes/clientes podem se sentir seguros (Scorsolini-Comin, 2015a).

Percebe-se, então, que a R/E é parte inegável da vida dos seres humanos e exerce influência na relação do sujeito com o mundo, seja a partir de suas próprias crenças ou por estar inserido em uma cultura que, independentemente, possui e promove características que são difundidas socialmente. Dessa maneira, se o profissional reconhece a própria $\mathrm{R} / \mathrm{E}$, permite-se aprender sobre os pacientes/clientes, lembrando que é preciso olhar a partir das contingências de cada cultura e indivíduo. Trata-se, também, de considerar e respeitar a expressão do paciente/cliente sem qualquer constrangimento, doutrinações ou induções para essa temática, o que está alinhado ao princípio de laicidade na atuação profissional (Scorsolini-Comin, 2015b; Silva \& Reichow, 2016) e em consonância com os preceitos éticos da atuação em Psicologia.

Embora alguns estudos coloquem como essencial o questionamento acerca da $\mathrm{R} / \mathrm{E}$ do paciente/cliente, por exemplo, durante a anamnese (Lucchetti et al., 2010), seguindo protocolos internacionais (Allmon, 2013), a maioria das produções destaca a necessidade de abrir espaço para essa dimensão, o que não equivale a incentivar que o relato seja orientado para a R/E. Mostrar-se aberto e poroso a essa temática significa estar preparado, com uma 
escuta que também pode se colocar à disposição de outras demandas e aspectos, a depender da queixa do cliente, sua história e evolução clínica. Assim, recomenda-se a escuta da $\mathrm{R} / \mathrm{E}$ como possibilidade, mas nem sempre como uma necessidade, o que irá depender do modo com que o próprio sujeito traz a sua narrativa e a conduz no espaço clínico. A próxima categoria irá abordar algumas estratégias descritas no corpus para promover essa escuta no espaço clínico, quando necessário.

\section{Categoria 3: R/E e Prática Clínica - Estratégias, Intervenções e Protocolos}

A R/E é um constructo multidimensional e pode ser articulado de formas diferentes pelos pacientes/clientes, sendo importante evitar suposições baseadas em afiliações religiosas ou culturais, pois mesmo com as mesmas tradições, diversos grupos podem apresentar práticas, interpretações e comportamentos diferentes (Moreira-Almeida, Koenig, \& Lucchetti, 2014). Em face dessa condição, a recomendação, muitas vezes, é a de que haja uma investigação e um levantamento particular do histórico espiritual/religioso de cada paciente/cliente.

O uso de ferramentas, ou a menção da possibilidade delas, pode ilustrar uma forma de se fazer esse levantamento. Uma das ferramentas disponíveis é o "FICA", que avalia a necessidade do paciente/cliente de trabalhar sua $\mathrm{R} / \mathrm{E}$ com base em questões voltadas para sua fé, a importância que tem para sua vida, a repercussão na comunidade e seu interesse em incluir o tema no tratamento (Leighton, 2014). Toma-se como referência, portanto, a decisão do paciente/ cliente de abordar essa dimensão em seu percurso ao longo da psicoterapia. Obviamente, o psicoterapeuta deve estar atento a esse aspecto e devidamente disponível e prenhe de recursos para essa inclusão.

Segundo o APA Ehtics Code Standard 2.01b, independentemente da abordagem, o profissional tem obrigação ética de inquirir o paciente/cliente a respeito de sua $\mathrm{R} / \mathrm{E}$, para contemplar e compreender fatores de diversidade na implementação de serviços, incluindo a avaliação de diagnóstico e interpretação de sintomas (Allmon, 2013). Essa avaliação não precisa ser nenhum processo complexo; no entanto, ferramentas estruturadas podem ser incorporadas por qualquer profissional da saúde, permitindo um reconhecimento da preocupação dos pacientes em relação à R/E (Richardson, 2014). Nesse sentido, alguns protocolos têm possibilitado, de fato, a inclusão dessa dimensão ao longo do tratamento, o que pode ocorrer de diferentes formas e em diversos níveis, até mesmo pela menção a esses aspectos e ao modo que as experiências espirituais/religiosas têm feito parte do repertório do indivíduo.

Em linha semelhante, na tentativa de ilustrar a possibilidade de incorporação da $\mathrm{R} / \mathrm{E}$ na prática, outros artigos também apresentaram a aplicação de ferramentas e procedimentos para trabalhar essa dimensão, como a "ASERVIC", que consiste em um guia instrumental de competências para trabalhar a R/E do cliente (Daniels \& Fitzpatrick, 2013). Existe também a "BRAIDS", uma ferramenta que estabelece, promove e mantém uma comunicação das crenças do sujeito (Leighton, 2014) e o "VITA", que é uma intervenção estruturada própria, intensiva, de 12 semanas. Outro recurso mencionado é "PRACTICE", um acrônimo específico usado na terapia cognitivo-comportamental para o trauma, o qual contém os passos necessários para realizar a intervenção e pode ser adaptado usando aspectos espirituais/religiosos (Walker et al., 2010). A técnica Calmer Life, um protocolo de tratamento cognitivo-comportamental para pacientes com transtorno de ansiedade generalizada, também foi apresentado com modificações que permitem a integração da $\mathrm{R} / \mathrm{E}$ de acordo com a preferência do paciente/cliente (Barrera et al., 2012). $\mathrm{O}$ uso dessas ferramentas de investigação e procedimentos de incorporação da $\mathrm{R} / \mathrm{E}$ revela a existência de protocolos que permitem o psicoterapeuta saber como proceder diante destas questões, ofertando um conhecimento mínimo e ético sobre o assunto, pois muitas vezes pode ser difícil e desafiador iniciar uma conversa a respeito disso (Richardson, 2014). Assim, o profissional pode instrumentalizar-se para possibilitar um diálogo mais aberto e direto sobre temáticas que se mostram, muitas vezes, de difícil manejo no espaço clínico.

Outras estratégias têm sido incorporadas pelos profissionais para lidar com questões espirituais/religiosas. Destaca-se o uso de recursos metafóricos ou esclarecimento de passagens bíblicas em pacientes/clientes fundamentalistas, de maneira a confrontar crenças e comportamentos sem ferilos, permitindo ampliar o conhecimento sobre si (Dein, 2013; Henning-Geronasso \& Moré, 2015; Hook et al., 2013). Jones (2014) utilizou a prece/reza como elemento de aproximação com uma paciente/cliente esquizofrênica, possibilitando a reformulação de sentidos e significados. Ao rezar junto com a paciente/cliente, $o$ autor permitiu que a mesma se sentisse acolhida pelo terapeuta, restabelecendo o vínculo entre eles. Nesse processo, a crença religiosa/espiritual da paciente/ cliente foi uma ponte para o trabalho terapêutico diante da avaliação de que era um aspecto importante para a mesma. Em alguns casos, o profissional pode encaminhar o paciente/ cliente para outro profissional mais indicado (por exemplo, padre ou pastor), devido à necessidade de aprofundamento específico sobre uma religião e, então, realizar um trabalho em parceria (Dein, 2013). Esses exemplos de intervenções também permitem o reconhecimento e a abertura para a $\mathrm{R} / \mathrm{E}$ na prática clínica e, para isso, não é necessário nenhum conhecimento específico ou aprofundamento sobre qualquer religião, tendo em vista que esse conhecimento possa existir a partir de uma investigação junto ao paciente/cliente.

O coping (enfrentamento) positivo se apresentou como uma forma de utilizar a R/E beneficamente em situações de dificuldades e, a partir disso, construir sentido diante delas, emergindo também a nomenclatura coping religioso/ 
espiritual (Karekla \& Constantinou, 2010; Lomax \& Carlin, 2015). A partir da expressão de que R/E é importante para paciente/cliente, o profissional pode usar suas crenças e comportamentos voltados para essa dimensão para lidar com situações de luto ou doenças graves, permitindo uma forma de entender, aceitar e enfrentar a dificuldade (Karekla \& Constantinou, 2010). Dessa forma, o coping religioso/ espiritual pode ser uma maneira de incorporar a $R / E$ na psicoterapia e, ainda, ajudar o paciente/cliente em seu processo de adaptação ao adoecimento (Richardson, 2014).

Práticas de meditação tais como mindfulness e conscious awareness também foram encontradas na revisão como formas de incorporar a R/E na prática clínica (Sperry, 2010; Zoysa, 2011). De acordo com Zoysa (2011) e Russell e Tatton-Ramos (2014), a meditação do tipo mindfulness é derivada do Budismo e tem por objetivo psicoterápico a regulação da atenção plena, o desenvolvimento de atitude de tolerância e a redução da evitação comportamental. Esse tipo de prática vem influenciando a terceira onda de terapias cognitivo comportamentais, como a Acceptance and Commitment Therapy (ACT), Dialectical Behavior Therapy (DBT) e a Mindfulness-Based Cognitive Therapy (MBCT), nas quais o paciente/cliente é estimulado a praticar a meditação e, depois, discutir os conhecimentos adquiridos. Já a conscious awareness é entendida como a consciência da existência, das sensações, dos pensamentos e dos sentimentos, que inclui sintonia e receptividade ao universo, em que níveis de awareness têm sido descritos pela literatura judaico-cristã como reflexo de desenvolvimento espiritual e atrelados também a abordagens mais cognitivo-comportamentais (Sperry, 2010). Práticas como o mindfulness são apresentados por estudos que comprovam seus benefícios, como a diminuição de transtornos de humor, alívio de estresse, maiores níveis de empatia, menores níveis de ansiedade. Além disso, demonstram confiança associativa (confiança, postura aberta e cuidado), motivação para unidade (sensação de ser parte de algo que é maior do que a própria pessoa) e aumento na experiência espiritual de estudantes de graduação (Snyder \& Lopez, 2009).

Todas essas estratégias e ferramentas sugerem uma variada possibilidade de se incorporar o conteúdo religioso e espiritual na prática sem reduzir o processo psicoterápico, legitimando essa dimensão na esfera científica. Entretanto, independentemente de uma incorporação diretamente trabalhada a partir de intervenções, técnicas e instrumentos, muitas pesquisas desta revisão apontam para a necessidade de fazer tudo isso com uma postura de respeito e acolhimento. Essas características são fundamentais, porque reconhecem a experiência do sujeito e acolhem e se interessam por este, favorecendo um vínculo terapêutico e permitindo a expressão de sentimentos negativos e possíveis distorções (Genaro Junior, 2011; Neubern, 2010a, 2010b; Oliveira \& Junges, 2012; Vandenberghe et al., 2012; Wade et al., 2014; Zenkert et al., 2014).

Assim, mais do que a busca por intervenções e protocolos, deve-se promover uma escuta aberta para questões envolvendo a $\mathrm{R} / \mathrm{E}$, de modo que as ferramentas descritas nessa categoria possam ser facilitadoras do processo, e não mecanismos diagnósticos ou que ofereçam um retrato avaliativo do sujeito. Tais ferramentas ou técnicas não excluem a necessidade de uma reflexão constante e densa sobre o tema, até mesmo para a tomada de decisão sobre atuar ou não na prática clínica. A discussão que se opera, portanto, é a de como esses recursos podem facilitar que a $\mathrm{R} / \mathrm{E}$, de fato, tenha espaço na prática clínica quando o sujeito considerar relevante e isso constituir uma demanda. Tais protocolos podem ser guias importantes para clínicos em cenários de consultórios ou mesmo em contextos como hospitais e serviços de saúde, em que nem sempre o setting é favorável à emergência de aspectos relacionados a R/E. Assim, recomenda-se o conhecimento de ferramentas e protocolos que possam ser empregados quando necessário, de modo crítico e em constante diálogo com as necessidades do paciente/cliente. Por fim, há que se ponderar que o mero emprego desses instrumentos descolados de uma reflexão mais densa não significa a incorporação da $\mathrm{R} / \mathrm{E}$ na prática clínica, mas sim apenas a consideração dessa dimensão como parte do processo diagnóstico ou do tratamento. Dessa forma, deflagra-se, com a presente revisão, a necessidade de não somente divulgar sugestões estratégicas recorrentes na literatura, mas também a de que estas sejam acompanhadas de sólidos apontamentos científicos em diálogo com o fazer clínico.

\section{CONSIDERAÇÕES FINAIS}

As publicações encontradas sugerem que os psicoterapeutas têm considerado a dimensão R/E integrada ao cuidado e à saúde, resguardando uma atenção à necessidade espiritual/religiosa do paciente/cliente. Essa visão possivelmente parte da percepção de que a $\mathrm{R} / \mathrm{E}$ constitui parte do processo cultural dos seres humanos, e que as especificidades derivadas dessa condição devem ser investigadas e incorporadas nas medidas de avaliação e diagnósticas, haja vista a influência que exerce sobre comportamentos e hábitos, sobre o bem-estar, na qualidade de vida e no manejo e enfrentamento de situações de saúde, de adoecimento e de outras dificuldades. Esse enquadramento multidimensional da $\mathrm{R} / \mathrm{E}$ em relação com a saúde e o componente cultural que esta oferece indicam um caminho para se introduzir a dimensão $\mathrm{R} / \mathrm{E}$ na prática.

O profissional também está sujeito a esse domínio e tem em si o dever de se posicionar sobre a sua própria $\mathrm{R} / \mathrm{E}$, mantendo um posicionamento de abertura para lidar 
com possíveis embates de opiniões e crenças, mas, acima de tudo, mantendo uma postura ética, com respeito e acolhimento. Alguns psicoterapeutas ainda relatam uma dificuldade ou desconhecimento de como proceder diante desse tema. Mesmo percebendo a importância que a $\mathrm{R} / \mathrm{E}$ possa ter em suas vidas e na dos pacientes/clientes, os psicoterapeutas podem se sentir inseguros e receosos em ferir alguma conduta ética. Essa dificuldade pode levar a uma postura de imparcialidade sobre o assunto. Isso sugere, mais uma vez, que discussões e reflexões a respeito de $\mathrm{R} / \mathrm{E}$ e suas interfaces devem permear a graduação dos profissionais, em formações complementares, grupos de estudo e supervisão, possibilitando a inserção natural do tema, menores incômodos e preconceito.

Estratégias e recursos desenvolvidos por terapeutas foram narrados na literatura e agregados às abordagens psicológicas de modo complementar, revelando uma variada instrumentalização para intervenções que considerem e incorporem a dimensão religiosa/espiritual do paciente/cliente, sobretudo no contexto internacional. Os psicoterapeutas acreditam que respeito e acolhimento são fundamentais na relação com pacientes/clientes, permitindo uma escuta verdadeira e a compreensão de suas necessidades. Analisando o corpus, sugere-se, principalmente aos profissionais brasileiros, maiores investigações dessa dimensão no que tange às suas experiências práticas e atuação psicoterápica, de maneira a fornecer subsídios para práticas éticas e inovadoras, superando as dificuldades enfrentadas por estes e, também, por recém-formados. Entrar em contato com essa literatura pode potencializar debates e reflexões importantes sobre o fazer profissional, em que se deve sempre considerar que não se trata de um tema de fácil abordagem e cujos achados estão em diálogo perene.

Em temos das limitações do presente trabalho, destaca-se a recuperação de estudos em apenas dois idiomas, ainda que se tenha evidenciado que a maioria da produção científica na área ocorra em língua inglesa. Ampliar o recorte temporal pode também ser um procedimento importante no sentido de localizar permanências e rupturas na produção científica das últimas décadas, trazendo importantes discussões para os pesquisadores da área. Sugere-se, ainda, que os cruzamentos incluídos no delineamento desta revisão também possam abarcar práticas terapêuticas desenvolvidas por profissionais de Psicologia que não apenas a psicoterapia, aliando práticas complementares e conhecimentos do aconselhamento multicultural, por exemplo, referido de modo recorrente nos estudos internacionais.

\section{REFERÊNCIAS}

Abu-Raiya, H. (2015). Working with religious muslim clients: A dynamic, qura'nic-based model of psychotherapy. Spirituality in Clinical Practice, 2(2), 120-133.

Abu-Raiya, H., \& Pargament, K. I (2010). Religiously integrated psychotherapy with muslim clients: From research to practice. Professional Psychology: Research and Practice, 41(2), 181188.

Alminhana, L. O., \& Moreira-Almeida, A. (2009). Personalidade e religiosidade/ espiritualidade $(\mathrm{R} / \mathrm{E})$. Archives of Clinical Psychiatry, 36(4), 153-161.

Allmon, A. L. (2013). Religion and the DSM: From pathology to possibilities. Journal of Religion and Health, 52, 538-549.

American Psychiatric Association - APA. (2014). Manual diagnóstico e estatístico de transtornos mentais - DSM V. (M. I. C. Nascimento et al., Trads.). Porto Alegre: Artmed.

Aten, J. D., Mangis, M. W., \& Campbell, C. (2010). Psychotherapy with rural religious fundamentalist clients. Journal of Clinical Psychology: In Session, 66(5), 513-523.

Bairrão, J. F. (2004). Espiritualidade brasileira e clínica psicológica. In V. A. Angerami-Camon (Org.), Espiritualidade e prática clínica (pp. 193-214). São Paulo: Thomson.

Barrera, T. L., Zeno, D., Bush, A. L., Barber, C. R., \& Stanley, M. A. (2012). Integrating religion and spirituality into treatment for late-life anxiety: Three case studies. Cognitive and Behavioral Practice, 19, 346-358.

Brown, O., Elkonin, D., \& Naicker, S. (2013). The use of religion and spirituality in psychotherapy: Enablers and barriers. Journal of Religion and Health, 52, 1131-1146.

Cavalheiro, C. M. F., \& Falcke, D. (2014). Espiritualidade na formação acadêmica em psicologia no Rio Grande do Sul. Estudos de Psicologia, 31(1), 35-44.

Carneiro, J. D. G. (2008). Experiência espiritual e psicopatologia: A percepção de professores de psicopatologia. Monografia de
Graduação em Psicologia, Universidade Católica de Brasília, Brasília, DF.

Ciclitira, K., \& Foster, N. (2012). Attention to culture and diversity in psychoanalytic trainings. British Journal of Psychotherapy, 28(3), 353-373.

Conselho Federal de Psicologia - CFP. (2013). Posicionamento do Sistema de Conselhos de Psicologia para a questão da Psicologia, religião e espiritualidade. GT Nacional Laicidade e Psicologia. Brasília: Autor.

Conselho Regional de Psicologia de São Paulo - CRP-SP. (2016). Laicidade, religião, direitos humanos e políticas públicas (Vol. 1). São Paulo: Autor.

Cornish, M. A., Wade, N. G., \& Knight, M. A. (2013). Understanding group therapists' use of spiritual and religious interventions in group therapy. International Journal of Group Psychotherapy, 63(4), 573-591.

Costa, C. C., Bastiani, M., Geyer, J. G., Calvetti, P. Ü., Muller, M. C., \& Moraes, M. L. A. (2008). Qualidade de vida e bemestar espiritual em universitários de Psicologia. Psicologia em Estudo, 13(2), 249-255.

Dal-Farra, R. A., \& Geremia, C. (2010). Educação em saúde e espiritualidade: Proposições metodológicas. Revista Brasileira de Educação Médica, 34(4), 587-597.

Dalgalarrondo, P. (2007). Estudos sobre religião e saúde mental realizados no Brasil: histórico e perspectivas atuais. Archives of Clinical Psychiatry, 34(1), 25-33.

Daniels, C., \& Fitzpatrick, M. (2013). Integrating spirituality into counselling and psychotherapy: Theoretical and clinical perspectives. Canadian Journal of Counselling and Psychotherapy, 47(3), 315-341.

Dein, S. (2013). Religious doubts: Implications for psychopathology and psychotherapy. Bulletin of the Menninger Clinic, 77(3), 201-221. 
Freitas, M. H. (2014). Religiosidade e saúde: Experiências dos pacientes e percepções dos profissionais. Revista Pistis e Práxis: Teologia e Pastoral, 6(1), 89-105.

Genaro Junior, F. (2011). Psicologia clínica e religiosidade/ espiritualidade: Interlocução relevante para a prática clínica contemporânea. Psicologia Revista, 20(1), 29-41.

Gottwald, C. (2014). Awareness and mindfulness in consciousnesscentered body psychotherapy. International Body Psychotherapy Journal, 13(1), 67-79.

Henning-Geronasso, M. C., \& Moré, C. L. (2015). Influência da religiosidade/espiritualidade no contexto psicoterapêutico. Psicologia: Ciência e Profissão, 35(3), 711-725.

Hill, P. C., Pargament, K. I, Hood, R. W., Jr., McCullough, M. E., Swyers, J. P., Larson, D. B., \& Zinnbauer, B. J. (2000). Conceptualizing religion and spirituality: Points of commonality, points of departure. Journal for the Theory of Social Behaviour, 30, 51-77.

Hook, J. N., Worthington Jr., E. L., Davis, D. E., \& Atkins, D. C. (2013). Religion and couple therapy: Description and preliminary outcome data. Psychology of Religion and Spirituality, 6(2), 94-101.

Jones, J. (2014). Spirituality as attachment: The psychotherapy treatment of a lesbian woman suffering from schizophrenia. Spirituality in Clinical Practice, 1(4), 307-312.

Karekla, M., \& Constantinou, M. (2010). Religious coping and cancer: Proposing an acceptance and commitment therapy approach. Cognitive and Behavioral Practice, 17, 371-381.

Keeling, M. L., Dolbin-MacNab, M. L., Ford, J., \& Perkins, S. N. (2010). Partners in the spiritual dance: Learning clients' steps while minding all our toes. Journal of Marital and Family Therapy, 36(2), 229-243.

Khodayarifard, M., \& Fatemi, S. M. (2013). Combination of spirituality and cognitive-behavioral family therapy on treatment of generalized anxiety disorder. Scientific Research, 4(4), 427-432.

Koenig, H. G. (2005). Espiritualidade no cuidado com o paciente. Por quê, como, quando e o quê. São Paulo: Editora FE.

Koenig, H. G. (2012). Religion, spirituality, and health: The research and clinical implications. ISRN Psychiatry, 2012.

Koenig, H. G., Berk, L. S., Daher, N. S., Pearce, M. J., Bellinger, D. L., Robins, C. J., Nelson, B., Shaw, S. F., Cohen, H. J., \& King, M. B. (2014). Religious involvement is associated with greater purpose, optimism, generosity and gratitude in persons with major depression and chronic medical illness. Journal of Psychosomatic Research, 77, 135-143.

Leighton, T. J. (2014). The therapeutics of counselling religious clients: Intake and assessment. International Journal of Mental Health and Addiction, 12, 295-311.

Lomax, J. W., \& Carlin, N. (2015). Utilizing religious and spiritual material in clinical care: Two cases of religious mourning. Spirituality in Clinical Practice, 3(1), 59-68.

Lucchetti, G., Granero, A. L., Bassi, R. M., Latorraca, R., \& Nacif, S. A. P. (2010). Espiritualidade na prática clínica: O que o clínico deve saber? Revista Brasileira de Clínica Médica, $8(2), 154-158$

Marques, L. F. (2010). O conceito de espiritualidade e sua interface com a religiosidade e a Psicologia Positiva. Revista Psicodebate Psicología, Cultura y Sociedad, 10, 135-151.

Mendes, K.D.S., Silveira, R. C. C. P., \& Galvão, C. M. (2008). Revisão integrativa: Método de pesquisa para a incorporação de evidências na saúde e na enfermagem. Texto \& Contexto Enfermagem, 17(4), 758-764.

Menezes, A., Jr., \& Moreira-Almeida, A. (2009). Differential diagnosis between spiritual experiences and mental disorders of religious content. Revista de Psiquiatria Clínica, 36, 75-82.
Moher, D., Liberati, A., Tetzlaff, J., Altman, D. G., \& The PRISMA Group (2009). Preferred reporting items for systematic reviews and meta-analyses: The PRISMA Statement. Annals of Internal Medicine, 151(4), 264-269.

Moreira-Almeida, A., Koenig, H. G., \& Lucchetti, G. (2014). Clinical implications of spirituality to mental health: Review of evidence and practical guidelines. Revista Brasileira de Psiquiatria, 36(2), 176-182.

Neubern, M. S. (2010a). Hipnose e subjetividade: Utilização da experiência religiosa em psicoterapia. Estudos de Psicologia, 27(2), 235-245.

Neubern, M. S. (2010b). Psicoterapia e religião: Construção de sentido e experiência do sagrado. Interação em Psicologia, 14(2), 263-273.

Oliveira, M. R., \& Junges, J. R. (2012). Saúde mental e religiosidade/ espiritualidade: A visão de psicólogos. Estudos de Psicologia, $17(3), 469-476$.

Paiva, G. J. (2005). Psicologia da religião, psicologia da espiritualidade: Oscilações conceituais de uma "disciplina". In M. M. Amatuzzi (Org.), Psicologia e espiritualidade (pp. 31-48). São Paulo: Paulus.

Panzini, R. G., \& Bandeira, D. R. (2007). Coping (enfrentamento) religioso/ espiritual. Archives of Clinical Psychiatry, 34(1), 126-135.

Panzini, R. G., Rocha, N. S., Bandeira, D. R., \& Fleck, M. P. A. (2007). Qualidade de vida e espiritualidade. Revista de Psiquiatria Clínica, 34(Supl. 1), 105-115.

Pargament, K. I. (2007). Spiritually integrated psychotherapy: Understanding and addressing the sacred. New York: The Guilford Press.

Richardson, P. (2014). Spirituality, religion and palliative care. Annals of Palliative Medicine, 3(3), 150-159.

Rich, M. L. (2012). Integrating shamanic methodology into the spirituality of addictions recovery work. International Journal of Mental Health Addiction, 10, 330-353.

Russell, T. A., \& Tatton-Ramos, T. P. (2014). Body in mind training: Mindful movement for the clinical setting. Neuro-Disability and Psychotherapy, 2, 108-136.

Santos, C. M. C., Pimenta, C. A. M., \& Nobre, M. R. C. (2007). A estratégia PICO para a construção da pergunta de pesquisa e busca de evidência. Revista Latino-Americana de Enfermagem, 15(3), 508-511.

Scorsolini-Comin, F. (2015a). Um toco e um divã: Reflexões sobre a espiritualidade na clínica etnopsicológica. Contextos Clínicos, 8(2), 114-127.

Scorsolini-Comin, F. (2015b). Elementos do aconselhamento multicultural aplicados à psicoterapia em contextos etnopsicológico. Estudos e Pesquisas em Psicologia, 15(2), 587-607.

Seligman, M. E. P. (2009). Felicidade autêntica: Usando a nova psicologia positiva para a realização permanente (N. Capelo, Trad.). Rio de Janeiro: Objetiva.

Silva, F. E., \& Reichow, J. R. C. (2016). Psicologia, laicidade e educação: Reflexões sobre a formação do psicólogo. In Conselho Regional de Psicologia de São Paulo, Laicidade, religião, direitos humanos e políticas públicas (Vol. 1, pp. 53-57). São Paulo: CRP-SP.

Smith, T. B., McCullough, M. E., \& Poll, J. (2003). Religiousness and depression: Evidence for a main effect and the moderating influence of stressful life events. Psychology Bulletin, 129(4), 614-636.

Souza, L. D. M., Maragalhoni, T. C., Quincoses, M. T., Jansen, K., Cruzeiro, A. L. S., Ores, L., Silva, R. A., \& Pinheiro, R. T. (2012). Bem-estar psicológico de jovens de 18 a 24 anos: Fatores associados. Cadernos de Saúde Pública, 28(6), $1167-$ 1174. 
Snyder, C. R., \& Lopez, S. J. (2009). Psicologia Positiva: Uma abordagem cientifica e prática das qualidades humanas $(\mathrm{R}$. C. Costa, Trad.). Porto Alegre: Artmed.

Sperry, L. (2010). Psychotherapy sensitive to spiritual issues: A postmaterialist psychology perspective and developmental approach. Psychology of Religion and Spirituality, 2(1), 46-56.

Stalsett, G., Engedal, L. G., \& Austad, A. (2010). The persecuting god and the crucified self: The case of Olav and the transformation of his pathological self-image. Pragmatic Case Studies in Psychotherapy, 6(2), 49-100.

Vandenberghe, L., Prado, F. C., \& Camargo, E. A. (2012). Spirituality and religion in psychotherapy: Views of Brazilian psychotherapists. International Perspectives in Psychology: Research, Practice, Consultation, 1(2), 79-93.

Wade, N. G., Post, B. C., Cornish, M. A., Vogel, D. L., \& Runyon-Weaver, D. (2014). Religion and spirituality in group psychotherapy: Clinical application and case example. Spirituality in Clinical Practice, 1(2), 133-144.

Walker, D. F., Reese, J.B., Hughes, J. P., \& Troskie, M. J. (2010). Addressing religious and spiritual issues in trauma-focused cognitive behavior therapy for children and adolescents. Professional Psychology: Research and Practice, 41(2), 174-180.

World Health Organization - WHO. (1998). WHOQOL and spirituality, religiousness and personal beliefs (SRPB) (General Report). Retirado do sítio da World Health Organization: https://apps.who.int/iris/bitstream/handle/10665/70897/ WHO_MSA_MHP 98.2_eng.pdf;jsessionid=4F39F64C487 642D5025930AA03FECĀ6B? sequence $=1$

Zajde, N. (2011). Psychotherapy with immigrant patients in France: An ethnopsychiatric perspective. Transcultural Psychiatry, 48(3), 187-204.

Zenkert, R. L., Brabender, V., \& Slater, C. (2014). Therapists' responses to religious/spiritual discussions with trauma versus non-trauma clients. Journal of Contemporary Psychotherapy, 44, 213-221.

Zoysa, P. (2011). The use of Buddhist mindfulness meditation in psychotherapy: A case report from Sri Lanka. Transcultural Psychiatry, 48(5), 675-683. 